\title{
High Average Power Mid-infrared Supercontinuum Generation in a Suspended Core Chalcogenide Fiber
}

\author{
Uffe Møller, ${ }^{1, *}$ Yi Yu, ${ }^{2}$ Christian R. Petersen, ${ }^{1}$ Irnis Kubat, ${ }^{1}$ Laurent Brilland, ${ }^{3}$ \\ David Méchin, ${ }^{3}$ Johann Troles, ${ }^{4}$ Barry Luther-Davies, ${ }^{2}$ and Ole Bang ${ }^{1,5}$ \\ ${ }^{1}$ DTU Fotonik, Department of Photonics Engineering, Technical University of Denmark, 2800 Kgs. Lyngby, Denmark \\ ${ }^{2}$ Centre for Ultrahigh bandwidth Devices for Optical Systems (CUDOS), Laser Physics Centre, \\ Australian National University, Canberra, ACT 0200, Australia \\ ${ }^{3}$ Perfos, R\&D Platform of Photonics Bretagne, 11 Rue Louis de Broglie, 22300 Lannion, France \\ ${ }^{4}$ Institut des Sciences, Chimiques de Rennes, Equipe Verres et Céramiques, Université de Rennes 1, \\ 35042 Rennes Cedex, France \\ ${ }^{5}$ NKT Photonics A/S, Blokken 84, 3460 Birkerød, Denmark \\ *ufmo@fotonik.dtu.dk
}

\begin{abstract}
Mid-infrared supercontinuum spanning from 2.0 to $6.1 \mu \mathrm{m}$ is generated in a $9 \mathrm{~cm}$ suspended core chalcogenide fiber by pumping close to the fiber zero-dispersion wavelength at $3.5 \mu \mathrm{m}$ with an OPA system.
\end{abstract}

OCIS codes: $320.6629,190.4370,160.4330,060.4005$.

\section{Introduction}

The mid-infrared spectral region is of great interest because a large number of molecules display fundamental vibrational transitions in this region, which leaves distinctive spectral fingerprints [1]. So far thermal sources, synchrotron sources and quantum cascade lasers (QCLs) have been the only possible mid-infrared light sources. However, thermal sources have low brightness, synchrotron sources are costly and not portable, and the tuning range of individual QCLs are limited and some wavelength ranges remain inaccessible. A mid-infrared supercontinuum light source will potentially offer high brightness, spatial coherence, broad spectral bandwidth, and portability, making it useful for applications involving medical diagnostics [2] and food analysis [3].

Supercontinuum light sources spanning the visible and near-infrared spectral region based on nonlinear effects in tailored photonic crystal fibers made of silica are commercial available. However, the material absorption of silica increases drastically when exceeding wavelengths of $2.4 \mu \mathrm{m}$ and silica can therefore not be used for efficient supercontinuum generation in the mid-infrared region. Several so-called soft-glass materials have been proposed as candidates for fibers suitable for the mid-infrared region, including tellurite, fluoride, heavy metal oxide, and chalcogenide glasses.

The chalcogenide glass $\mathrm{As}_{2} \mathrm{Se}_{3}$ transmits light out to $17.5 \mu \mathrm{m}$ [4] and fibers made of $\mathrm{As}_{2} \mathrm{Se}_{3}$ possess strong optical nonlinearities up to 1,000 times greater than that of silica fibers [5]. However, the use of such materials for supercontinuum generation has so far been limited by the lack of high peak power pump sources in the mid-infrared region.

Here we show mid-infrared supercontinuum generation from 2.0 to $6.1 \mu \mathrm{m}$ in a suspended core $\mathrm{As}_{2} \mathrm{Se}_{3}$ fiber by pumping with $320 \mathrm{fs}$ pulses at $3.5 \mu \mathrm{m}$ from an optical parametric amplifier (OPA) system.

\section{Experimental Results}

For our experiments we used an $\mathrm{As}_{2} \mathrm{Se}_{3}$ suspended core fiber provided by Perfos which had a core diameter of $4.5 \mu \mathrm{m}$ and was similar to the ones presented in [6]. The material loss (Fig. 1(a), blue line) was as low as $1 \mathrm{~dB} / \mathrm{m}$ around $5 \mu \mathrm{m}$ and below $4 \mathrm{~dB} / \mathrm{m}$ from 1.5 to $8.2 \mu \mathrm{m}$, except for the peaks at $3 \mu \mathrm{m}$ and $4.3 \mu \mathrm{m}$, which were due to O-H and C-O absorption, respectively. The calculated fiber dispersion shown in Fig. 1(a) (black line) was based on the suspended core structure shown in the inset of Fig. 1(a), and the zero-dispersion wavelength (ZDW) was calculated to be $3.52 \mu \mathrm{m}$.

A $9 \mathrm{~cm}$ fiber was pumped with an OPA system delivering $320 \mathrm{fs}$ pulses at $3.5 \mu \mathrm{m}$ with a repetition rate of $21 \mathrm{MHz}$ [7]. The pulse peak power was approximately $15 \mathrm{~kW}$ per $100 \mathrm{~mW}$ of average power. A molded chalcogenide lens with an NA of 0.85 was used to couple the light to the fiber, while reflective optics was used to collect 
the light after the fiber and guide it to a Newport Cornerstone $0.25 \mathrm{~m}$ monochromator. PbSe and MCT detectors were used to measure the spectra in the range 1.4-4.0 $\mu \mathrm{m}$ and 4.0-7.0 $\mu \mathrm{m}$, respectively. The total insertion loss to the fiber including input lens and output optics, any overlap loss and any propagation losses was measured to be $7 \mathrm{~dB}$, and thus the power delivered to the fiber was estimated to be $3.5 \mathrm{~dB}$ lower than the laser output, i.e. $6.6 \mathrm{~kW}$ of peak power per $100 \mathrm{~mW}$ of average power.
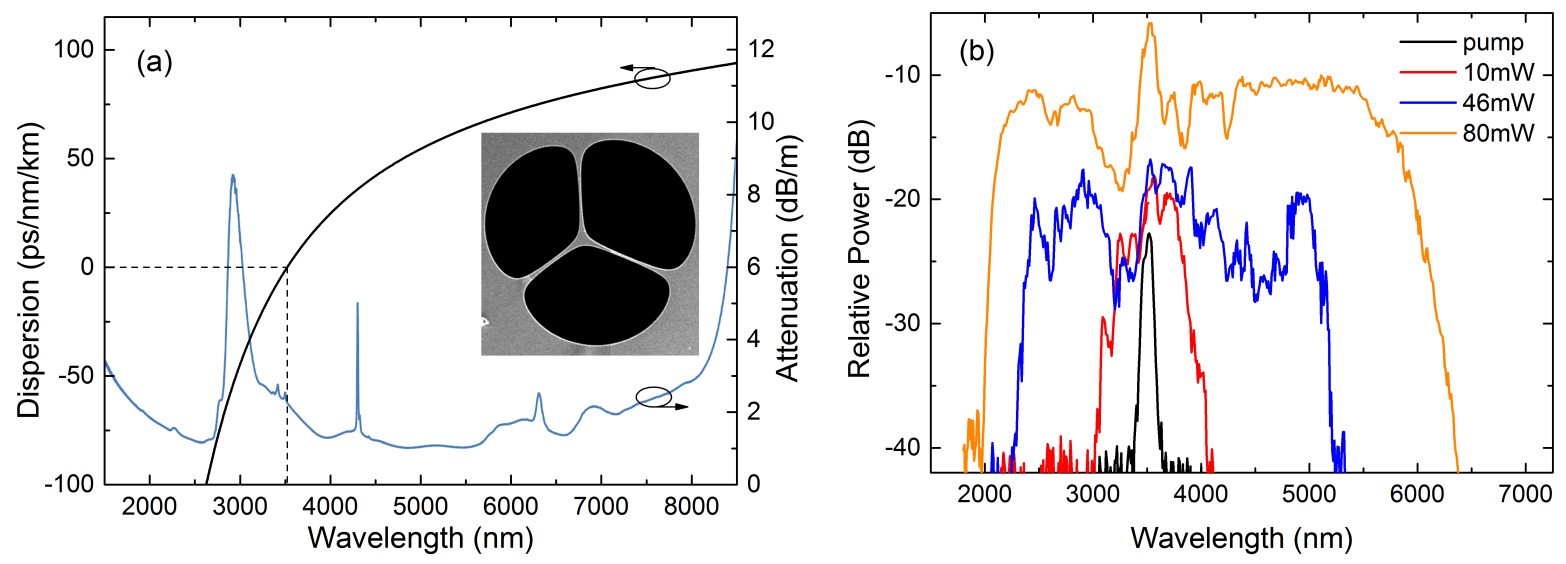

Fig. 1. (a) Calculated dispersion and measured material loss. Inset: SEM image of the fiber with a core diameter of $4.5 \mu \mathrm{m}$. (b) Output spectra measured as a function of input power.

Spectra as a function of the laser average power are shown in Fig. 1(b). Pumping close to the ZDW of the fiber will lead to soliton and dispersive wave formation in the anomalous and normal dispersion regime, respectively. The solitons propagating in the fiber will redshift due to the effect of soliton self-frequency shifting [8], while the dispersive waves will blueshift due to being trapped by group-velocity matched solitons [9]. High power solitons will redshift more compared to low power solitions and as a consequence the spectrum will broaden when increasing the input power. Due to the short piece of fiber the absorption peaks at $3 \mu \mathrm{m}$ and $4.3 \mu \mathrm{m}$ is of little significance to the spectral broadening, however dips in the spectra at these wavelengths are observed. In this case the broadest spectrum obtained spans from 2.0 to $6.1 \mu \mathrm{m}$ measured at the $-20 \mathrm{~dB}$ level with an average output power of $16 \mathrm{~mW}$.

Furthermore, we will in this presentation show the results of varying pump wavelength, fiber length, and pump power, respectively, and we will discuss how this affects the bandwidth of the generated supercontinuum.

\section{Conclusion}

We have demonstrated supercontinuum generation in a $9 \mathrm{~cm} \mathrm{As}_{2} \mathrm{Se}_{3}$ suspended core fiber, which was pumped with an OPA system delivering $320 \mathrm{fs}$ pulses at $3.5 \mu \mathrm{m}$ with a repetition rate of $21 \mathrm{MHz}$. The broadest generated spectrum was spanning from 2.0 to $6.1 \mu \mathrm{m}$ measured at the $-20 \mathrm{~dB}$ level. For higher input power or longer pieces of fibers broader supercontinua may be generated.

\section{References}

1. A. Schliesser, N. Picqué, and T. W. Hänsch, "Mid-infrared frequency combs," Nat. Photonics 6, 440-449 (2012).

2. R. W. Waynant, I. K. Ilev, and I. Gannot, "Midinfrared laser applications in medicine and biology," Phil. Trans. R. Soc. Lond. A 359, 635-644 (2001).

3. J. Wegener, R. H. Wilson, and H. S. Tapp, "Mid-infrared spectroscopy for food analysis : recent new applications and relevant developments in sample presentation methods,", Trends Anal. Chem. 18, 85-93 (1999).

4. V. S. Shiryaev and M. F. Churbanov, "Trends and prospects for development of chalcogenide fibers for mid-infrared transmission," J. Non. Cryst. Solids 377, 225-230 (2013).

5. B. J. Eggleton, B. Luther-Davies, and K. Richardson, "Chalcogenide photonics," Nat. Photonics 5, 725-725 (2011).

6. J. Troles, Q. Coulombier, G. Canat, M. Duhant, W. Renard, P. Toupin, L. Calvez, G. Renversez, F. Smektala, M. El Amraoui, J. L. Adam, T. Chartier, D. Mechin, and L. Brilland, "Low loss microstructured chalcogenide fibers for large non linear effects at $1995 \mathrm{~nm}$," Opt. Express 18, 2664754 (2010).

7. Y. Yu, X. Gai, P. Ma, D.-Y. Choi, Z. Yang, R. Wang, S. Debbarma, S. J. Madden, and B. Luther-Davies, "A stable, broadband, quasi-continuous, mid-infrared supercontinuum generated in a chalcogenide glass waveguide," Laser Photonics Rev., accepted (2014).

8. J. M. Dudley, G. Genty, and S. Coen, "Supercontinuum generation in photonic crystal fiber," Rev. Mod. Phys. 78, 1135-1184 (2006).

9. A. V. Gorbach and D. V. Skryabin, "Light trapping in gravity-like potentials and expansion of supercontinuum spectra in photonic-crystal fibres," Nat. Photonics 1, 653-657 (2007) 